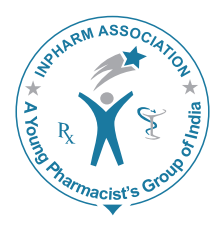

\title{
Design and evaluation of solid self-emulsifying drug delivery system of rosuvastatin calcium
}

\author{
Vipul Rokad ${ }^{1}$, Chirag Nagda ${ }^{2 *}$, Dhruti Nagda ${ }^{1}$ \\ Department of Pharmaceutics, ${ }^{I}$ A. R. College of Pharmacy \& G. H. Patel Institute of Pharmacy, Vallabh \\ Vidyanagar, Anand, Gujarat, India, ${ }^{2}$ Indukaka Ipcowala College of Pharmacy, New Vallabh Vidyanagar, \\ Anand, Gujarat, India
}

\begin{abstract}
Background: The work has been undertaken with an objective to prepare a self-emulsifying drug delivery system (SEDDS) of rosuvastatin calcium (ROS) with the least amount of surfactant which could enhance its solubility and oral bioavailability. Materials and Methods: Spectrophotometric method was used to estimate the solubility of the drug in various oils, surfactant and co-surfactant. The phase behavior and most efficient self-emulsifying region were identified by constructing pseudo ternary phase diagram. The solid-SEDDS (S-SEDDS) was prepared using an adsorbent consequently the prepared S-SEDDS were filled up in hard gelatin capsule which were evaluated for various physicochemical parameters. Results: The composition of optimized formulation consisted of $10 \mathrm{mg}$ of ROS, capmul MCM (oil), cremophor ELP (surfactant) and propylene glycol (co-surfactant). The optimized formulation showed negative zeta potential $(-22.11 \mathrm{mv})$, optimal particle size $(10.59 \mathrm{~nm})$ and demonstrated excellent self-emulsifying ability with a maximum solubilizing capability. The S-SEDDS formulations were prepared from the optimized liquid SEDDS, which revealed maximum release rate (97.7\%) among all the prepared S-SEDDS formulation and marketed formulation. Conclusion: It was concluded that S-SEDDS prepared from liquid SEDDS can be a promising novel approach to enhance the solubility and drug release of ROS, which in turn also develops a stable formulation, least drugs leakage and precipitation than the liquid SEDDS.
\end{abstract}

Key words: Adsorbent, bioavailability, drug release, rosuvastatin calcium, self-emulsifying drug delivery system, solubility

\section{INTRODUCTION}

For the treatment of many chronic diseases, oral route has been a major route for drug delivery. The oral

\begin{tabular}{|c|c|}
\hline \multicolumn{2}{|c|}{ Access this article online } \\
\hline Journal Sponsor & \multirow[b]{2}{*}{$\begin{array}{l}\text { Website: } \\
\text { www.jyoungpharm.org }\end{array}$} \\
\hline \multirow{2}{*}{ www.phcog net } & \\
\hline & $\begin{array}{l}\text { DOI: } \\
\text { 10.5530/jyp.2014.3.7 }\end{array}$ \\
\hline
\end{tabular}

delivery of many new drug candidates is limited due to its poor aqueous solubility and delivery of such drugs is recurrently associated with low oral bioavailability, increased inter and intra-subject variability which in turn leads to lack of dose proportionality. These problems can be overcome by various drug delivery strategies which include size reduction, formation of salt, $\beta$-cyclodextrins, nanoparticulate systems and solid dispersions. Further, these approaches utilize surfactants, lipids, permeation enhancers. ${ }^{1,2}$ In recent times, the lipid-based formulations have gained much attention and most prominence is self-emulsifying drug delivery systems (SEDDS), which

*Address for correspondence:

Dr. Chirag Nagda, Department of Pharmaceutics, Indukaka Ipcowala College of Pharmacy, New Vallabh Vidyanagar, Anand - 388 121,

Gujarat, India.E-mail: cnagda_rx@rediffmail.com 
helps to improve the oral bioavailability of non-aqueous drugs. 3,4

SEDDS are homogenous dispersion consisting of solid or liquid surfactants, synthetic or natural oils alternatively either co-solvents/surfactants and one or more hydrophilic solvents. These systems can prepare fine oil-in-water $(\mathrm{o} / \mathrm{w})$ emulsions or microemulsions upon mild agitation subsequent dilution in aqueous media, i.e., gastrointestinal (GI) fluids. ${ }^{2,5}$ The frequent irritation can arise because of prolonged contact between drug substance and the gut wall which could be minimized by fine oil droplets of SEDDS which would pass rapidly from the stomach and promote wide distribution of the drug throughout the GI tract. ${ }^{3}$ The SEDDS formulations are physically stable and easy to manufacture when compared to emulsions, which are metastable dispersion formulation. A large interfacial area for partitioning of the drug between oil and water is provided by SEDDS formulations, which are an added advantage over simple oily solutions. ${ }^{4}$

Conventionally a soft gelatin capsules are used to administer SEDDS which are usually prepared as a liquid dosage form, but it has certain limitations particularly related to the manufacturing process and incompatibility among the soft gelatin shells. ${ }^{6}$ One the technique to prepare solid SEDDS (S-SEDDS) is the use of solid carriers as an adsorbent. The formulation is adsorbed by a solid carrier and subsequently free flowing powders may be obtained from liquid self-emulsifying preparation. This S-SEDDS can be prepared by a simple process which involves the addition of liquid SEDDS to a carrier in a mixing blender and resulting the powder is directly filled into capsules. One of the advantages of an adsorbent technique is to obtain a good uniformity of content. ${ }^{7,8}$

A synthetic lipid-lowering agent, rosuvastatin calcium (ROS) is potent, selective and inhibits competitively to a 3-hydroxy-3-methylglutaryl-coenzyme. A reductase, which is used in the treatment of mixed dyslipidemia, primary hypercholesterolemia and hypertriglyceridemia. The site of absorption for ROS is stomach, and it is very less soluble in aqueous media with $20 \%$ absolute bioavailability. ${ }^{9,10}$ We hypothesized that S-SMEDDS encompassing ROS could be a vital strategy in enhance its solubility and drug release rate. Therefore, the aim of the present study is to prepare and evaluate S-SEDDS formulations to enhance the dissolution rate and bioavailability of ROS.

\section{MATERIALS AND METHODS}

\section{Materials}

ROS was obtained as a gift sample from Pravah Lab Pvt., Ltd., Hyderabad, India. Capmul MCM, capmul MCM PG8, capmul GMO, captex 200 P, captex 355 were a generous gift from Abitec Corporation, USA. Labrasol, Labrafec PG, Labrafil 1944 CS, captex 200 and capryol 90 were graciously donated by Gattefosse, France. Miglyol 810, miglyol 812 and miglyol 840 was obtained as gift sample from Saslo, Germany. Cremophor ELP and cremophore RH40 were brought from BASF, Germany. Polyethylene glycol 400 and propylene glycol were generous gifts from Allied Chemical Corporation, Vadodara, India. Tween 80, tween 20, span 80 and span 20 were procured from SD Fine Chemicals, Vadodara, India. Aerosil was purchased from Chemdyes Corporation, Ahmedabad, India. All chemicals were of analytical or technical grade and were used without further treatment.

\section{Methodology}

\section{Solubility determination of ROS}

In order to determine the suitable oils for preparation of SEDDS the solubility of ROS was calculated in 11 different oils by addition an excess amount of drug in $1 \mathrm{ml}$ of selected oils (capmul MCM, capmul MCM PG8, capmul GMO, miglyol 810, miglyol 812, miglyol 840, Labrafec PG, Labrafil 1944 CS, captex 200, captex 200 P, captex 355) and subsequently the distilled water was added separately in $2 \mathrm{ml}$ capacity microtube which were than mixed in a vortex mixer for $10 \mathrm{~min}$. The vials containing mixture were set aside for $72 \mathrm{~h}$ at room temperature to reach equilibrium. After equilibrium, each sample was centrifuged at $3000 \mathrm{rpm}$ for $15 \mathrm{~min}$. Sequentially, supernant was passed through membrane filter using $0.45 \mu \mathrm{m}$ filtered disc. ${ }^{11}$ Subsequently, the amount of ROS was calculated form supernatant by ultra violet (UV) spectrophotometer at $244 \mathrm{~nm} .^{12}$

\section{Construction of pseudoternary phase diagram}

The different weight ratios (1:1, 2:1 and 3:1) of surfactant and co-surfactant (Smix) in each group were mixed and the increasing concentration of surfactant with reference to cosurfactant was selected as Smix ratios. From the results of the solubility, capmul MCM (as the oil phase), cremophor ELP (as surfactant) and propylene glycol (as co-surfactant) were selected. The pseudo ternary phase diagram were constructed from Smix ratio and selected oil which was thoroughly mixed in varying mass ratios from 1:9 to 9:1 in stopper glass vials. The homogenous and transparent mixture

Journal of Young Pharmacists Vol 6 • Issue 3 • Jul-Sep 2014 
of oil and S/CoS was formed after vortexing it for $5 \mathrm{~min}$. After titration with water, the each mixture was visually observed for flow ability and phase clarity. The amount of water was recorded at which the phase transition occurred. The boundaries of the microemulsion domain were determined from this obtained values where analogous to the selected value of oils and the $\mathrm{S} / \mathrm{CoS}$ mixing ratio. ${ }^{13}$ The oil phase consisting of drug was used to study the effect of the drug on the construction of microemulsion boundary and phase diagram. CHEMIX-School 3.51 software was used to construct the phase diagrams. ${ }^{14}$

\section{Preparation of SEDDS formulations}

The various SEDDS formulations were prepared as per the composition depicted in Table 1. All the batches were prepared using $10 \mathrm{mg}$ of ROS, and the amount of SEDDS selected such that it solubilizes all the amount of drug completely. The drug was added to the mixture sequentially vortex mixture was to mix it completely at $37^{\circ} \mathrm{C}$. The prepared formulation was stored at room temperature until used. ${ }^{13,14}$

\section{Preparation of S-SEDDS}

S-SEDDS were prepared by use of Aerosil 200 (amorphous colloidal silicon dioxide) as an adsorbent to load ROS SEDDS (R-SEDDS). ${ }^{8}$ The addition of lipid formulations were gradually increased and mixed with the adsorbent which followed with predetermined G-SEDDS to adsorbent by weight ratio: $0.25: 1,0.5: 1,0.75: 1,1: 1,1.25: 1$, 1.5:1, 2:1, 2.25:1, 2.5:1, 3:1, 3.25:1. The mixture was than mixed in a mortar and initially a same amount of R-SEDDS was added. The addition was continued until a poor flowing cohesive mass is formed. ${ }^{10}$ Among all the different ratios, 2.5:1 form free flowing granules was selected for solidification of R-SEDDS.

Table 1: Formulations of rosuvastatin calcium SEDDS

\begin{tabular}{lccccc}
\hline $\begin{array}{l}\text { Batch } \\
\text { no. }\end{array}$ & $\begin{array}{c}\text { Drug } \\
(\mathbf{m g})\end{array}$ & $\begin{array}{c}\text { Oil } \\
\%\end{array}$ & $\begin{array}{c}\text { Surfactant } \\
\%\end{array}$ & $\begin{array}{c}\text { Cosurfactant } \\
\%\end{array}$ & Smix \\
\hline $\mathrm{F}_{1}$ & 10 & 10 & 45 & 45 & $1: 1$ \\
$\mathrm{~F}_{2}$ & 10 & 15 & 42.5 & 42.5 & $1: 1$ \\
$\mathrm{~F}_{3}$ & 10 & 20 & 40 & 40 & $1: 1$ \\
$\mathrm{~F}_{4}$ & 10 & 25 & 32.5 & 32.5 & $1: 1$ \\
$\mathrm{~F}_{5}$ & 10 & 30 & 35 & 35 & $1: 1$ \\
$\mathrm{~F}_{6}$ & 10 & 10 & 60 & 30 & $2: 1$ \\
$\mathrm{~F}_{7}$ & 10 & 15 & 56.6 & 28.3 & $2: 1$ \\
$\mathrm{~F}_{8}$ & 10 & 20 & 53.3 & 26.6 & $2: 1$ \\
$\mathrm{~F}_{9}$ & 10 & 25 & 50 & 25 & $2: 1$ \\
$\mathrm{~F}_{10}$ & 10 & 30 & 46.6 & 23.3 & $2: 1$ \\
$\mathrm{~F}_{11}$ & 10 & 10 & 67.5 & 22.5 & $3: 1$ \\
$\mathrm{~F}_{12}$ & 10 & 15 & 63.7 & 21.3 & $3: 1$ \\
$\mathrm{~F}_{13}$ & 10 & 20 & 60 & 20 & $3: 1$ \\
$\mathrm{~F}_{14}$ & 10 & 25 & 56.2 & 18.7 & $3: 1$ \\
$\mathrm{~F}_{15}$ & 10 & 30 & 52.5 & 17.75 & $3: 1$ \\
\hline
\end{tabular}

Journal of Young Pharmacists Vol 6 • Issue 3 • Jul-Sep 2014

\section{Characterization of SEDDS formulation ${ }^{15,16}$}

\section{Appearance}

Visual clarity of the prepared 15 formulations (F1-F15) was performed against black and white background and further the turbidity of batches was verified as per the method described by Singh $e t a l .^{16}$

\section{Refractive index}

The clarity of prepared batches could be used to access the refractive index. It was determined using Abbes Refractometer (1310 E-20 Atago, Japan) and distilled water was employed as standard.

pH

$\mathrm{pH}$ values of SEDDS were determine using $\mathrm{pH}$ meter $(\mu \mathrm{pH}$ system 302, Systronic, Ahmedabad). The calibration of $\mathrm{pH}$ meter was performed using buffer tablets of $\mathrm{pH} 4$ and $\mathrm{pH} 7$.

\section{Conductance}

Conductivity measurement could be used to predict the type of emulsion (either $\mathrm{o} / \mathrm{w}$ or w/o). The electro conductometer (314 Systronics) was used to determine the conductivity of the prepared formulations. Firstly, the electrodes were calibrated using $0.1 \mathrm{~N} \mathrm{KCl}$ (conductivity of $15.39 \mathrm{~ms} / \mathrm{cm}$ at $35^{\circ} \mathrm{C}$ ). Subsequently, conductivity of prepared microemulsion was measured in a $0.1 \mathrm{~N} \mathrm{NaCl}$ aqueous solution.

\section{\% Transmittance}

The percentage transmittance of prepared SEDDS were determined at $650 \mathrm{~nm}$ using UV spectrophotometer (UV1601, Shimadzu Corporation, Japan) keeping distilled water as blank.

\section{Particle size distribution and zeta potential analysis}

For the determination of droplet size and zeta potential the prepared formulations of SEDDS were suitably diluted with distilled water. To ensure complete dispersion of the formulation, the samples were inverted twice. Following complete dispersion, the microemulsions were subjected to Zetasizer (Nano ZS, Malvern Instruments, UK) for the droplet size determination. The principle involved is due to Brownian motion of droplets as a function of time which is determined due to fluctuation in light scattering, and it determines by photon correlation spectroscopy.

\section{Transmission electron microscopy (TEM)}

The selected batch of microsemuslion was used for TEM determination (Tecani, G2 20 TEM; Philips, Holland). The sample was kept on carbon coated grid $(3 \mathrm{~mm})$ which was set aside for dying. To obtain more contrast between the oil phase and the aqueous phase from the sample, 
$1 \%$ phosphotungustic acid solution was added as negative solution and these was dried in air for $2 \mathrm{~min}$. Afterwards, the samples were placed in horizontal sample holder of TEM. The magnification up to $\times 1600$ was used at $300 \mathrm{KV}$ acceleration voltage and images were taken.

\section{Characterization of S-SEDDS}

\section{Visual observation}

The visual observation of the diluted SEDDS was performed as per the method described by Singh et al. ${ }^{16}$ The emulsification parameters of prepared formulations were determined by placing the samples in $100 \mathrm{ml}$ of distilled water and stirred manually at room temperature. If the formulations remained as transparent, it is referred to as good and if it remained as milky or poor than it is denoted as bad. The microemulsions were observed for $24 \mathrm{~h}$ to check any sign of drug precipitation.

\section{Robustness on dilution}

For the determination of robustness to dilution the prepared formulations were diluted to 10, 100 and 1000 times using various media, i.e., distilled water, phosphate buffer $\mathrm{pH} 6.8$ and $0.1 \mathrm{~N} \mathrm{HCl}$. Than these samples were observed for drug precipitation or phase separation during the period of $12 \mathrm{~h} .{ }^{17}$

\section{Drug content}

The drug content of prepared formulations was found out by accurately weighing sample and dissolving it in $10 \mathrm{ml}$ of methanol. Further, the solution was filtered using Whatman filter paper, and the amount was estimated at $244 \mathrm{~nm}$ by the UV spectrophotometer (UV-1601 Shimadzu Corporation, Japan). ${ }^{6,12}$

\section{Differential scanning colorimetry (DSC)}

The quantitative and qualitative information regarding the physical state of ROS could be determined by studying thermal properties of S-SEDDS using DSC. The accurately weighed samples sealed in aluminum pans were heated in the temperature range of $50-230^{\circ} \mathrm{C}$ at a rate of $10^{\circ} \mathrm{C} / \mathrm{min}$ and nitrogen gas as an inert atmosphere was purged at flow rate of $50 \mathrm{ml} / \mathrm{min}$. The thermograms of ROS, aerosil and selected batch of S-SEDDS were recorded on DSC Pyris-1 (Perkin Elmer Instrument) and were compared to study any interaction with drug and excipients.

\section{Fourier transform infrared (FTIR) study}

An FTIR spectrum of the formulation was recorded on a spectrum GX-FTIR system (Perkin Elmer USA). A pellet of the drug in an isotropic mixture and other excipients were prepared with potassium bromide (spectroscopic grade) using hydraulic pellet press at a pressure of
7-10 tones. The spectrum was scanned in the range of $400-4000 / \mathrm{cm}$ while keeping the resolution of $1 / \mathrm{cm}$.

\section{In vitro dissolution studies}

In vitro dissolution study of all the prepared formulations consisting of $10 \mathrm{mg}$ ROS were performed using Type II dissolution apparatus (Veego Scientific USP Standard DA60). ${ }^{18}$ The prepared formulations were filled in hard gelatin capsules and placed in a dissolution vessels containing $900 \mathrm{ml}$ of phosphate buffer $\mathrm{pH} 6.8$ maintained at $37 \pm 0.5^{\circ} \mathrm{C}$, further the paddle speed was set at 50 RPM. After the time interval of $5,10,15$ and $20 \mathrm{~min}$, the samples $(5 \mathrm{ml})$ were withdrawn, and the fresh medium was added to replace the withdrawn sample. Furthermore, the samples were diluted suitably, and the amount of ROS was determined using a spectrophotometric method at $240 \mathrm{~nm}$ (UV-1601 Shimadzu corporation, Japan). ${ }^{12}$ Similarly, drug release study was performed for marketed product for comparison with the prepared formulation.

\section{RESULTS AND DISCUSSION}

\section{Solubility determination of ROS}

The ability of SEDDS formulation to retain solubility of the drug in the oil phase is significantly affected by the solubility of it in the oil phase. ${ }^{16}$ Therefore, the solubility of ROS was determined in various oil, surfactant and co-surfactant. The results of solubility study of ROS in various oils are depicted in Figure 1. It can be observed from the results that the highest solubility of ROS was in capmul MCM. The selection of a suitable surfactant may rely on the amount of surfactant because higher amount of it may lead to GI irritation. Further, it has been reported that ionic surfactants are more toxic than non-ionic surfactant because these may have lower value of critical micelle concentration as compared to ionic surfactant. The stable SEDDS could be prepared using mix of high and low surfactant which remains stable after dilution with water. ${ }^{5}$ The amount of this mix could be decreased using co-surfactant, which helps to make a flexible interfacial film of the curvatures required to prepare a stable microemulsion over any composition range. ${ }^{19}$ It can be observed from Figure 1 that satisfactory solubility of ROS was obtained in Tween 80, cremophor ELP, PEG 400 and PG. From the results of solubility study, cremophor ELP as surfactant and propylene glycol as co-surfactant was selected for further studies.

\section{Construction of pseudoternary phase diagram}

The stable formulations can be selected from the ternary phase diagram in the least possible time. An optimum formula of the R-SEDDS can be obtained from a microemulsion region upon dilution with water. The water

Journal of Young Pharmacists Vol 6 • Issue 3 • Jul-Sep 2014 


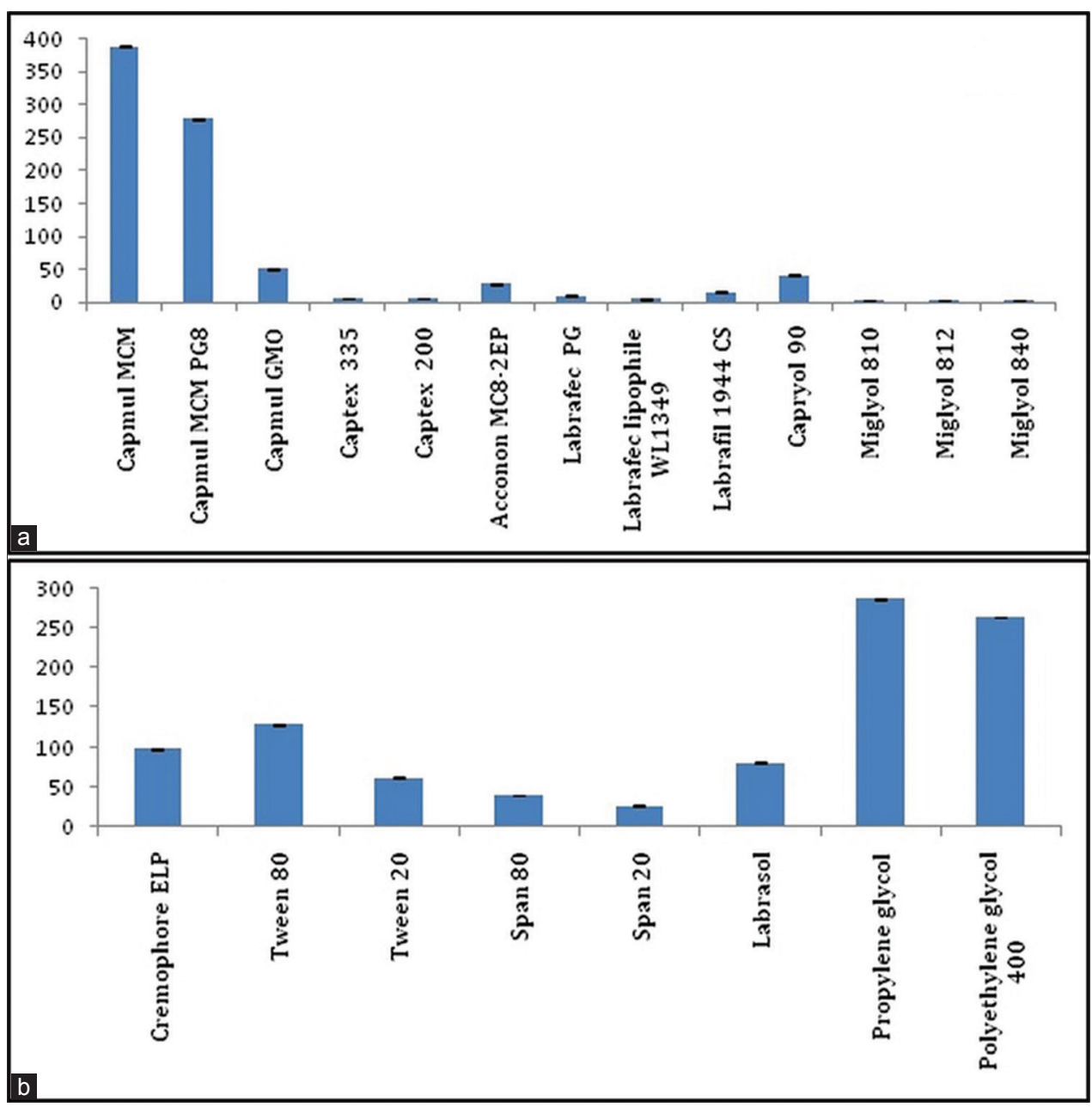

Figure 1: (a) Solubility of rosuvastatin calcium in various oils and (b) solubility of rosuvastatin calcium in various surfactants and cosurfactants

titration method was employed to plot the pseudoternary phase diagram of oil and surfactant/co-surfactant (S/CoS). The pseudoternary phase diagrams consisting of capmul MCM, cremophor ELP and PG system are shown in Figure 2. The following components were selected for the phase diagram: Capmul MCM (oil), cremophor ELP (surfactant) and Propylene glycol (co-surfactant). It can be seen from the plot that microemulsion region is in blue color whereas non-shaded areas display the turbid region based on visual observation. The microemulsion region was increased with increase in concentration of surfactant as compare to co-surfactant. Various other investigators have also documented that the use of high concentration of surfactants is necessary for these combinations to achieve fast and efficient self-emulsification. ${ }^{16,20}$

\section{Characterization of SEDDS formulation}

\section{Appearance}

The appearance, i.e., clarity, precipitation of drug and phase separation of the prepared formulations was observed for

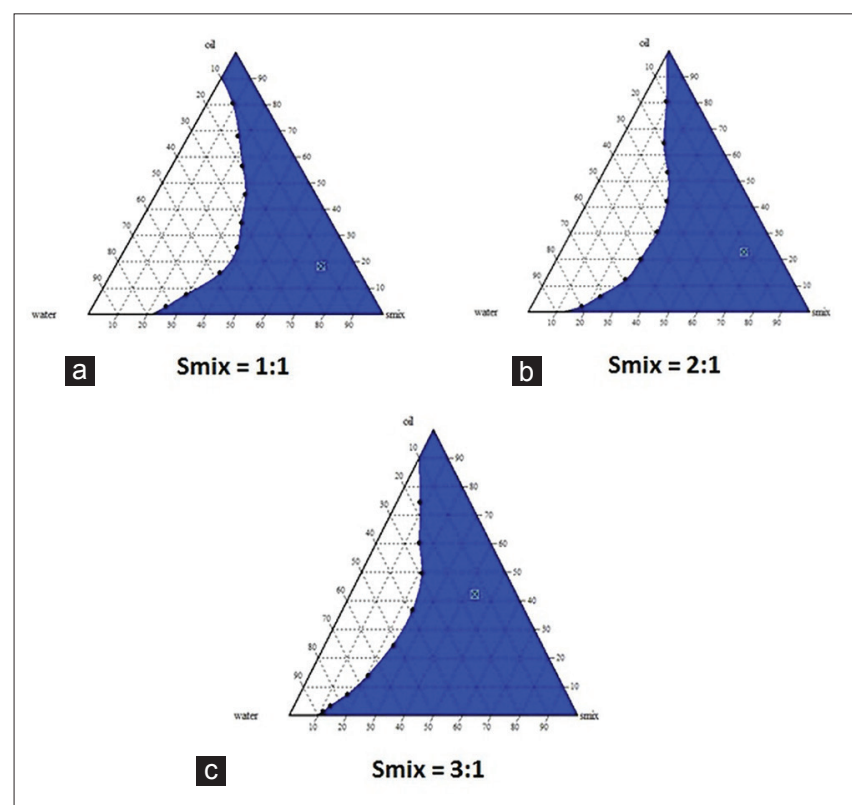

Figure 2: (a-c) Pseudoternary phase diagram of self-emulsifying drug delivery system containing capmul MCM, cremophor ELP, PG with Smix ratio $1: 1,2: 1,3: 1$ respectively 
$24 \mathrm{~h}$. The prepared formulations were denoted as stable (no precipitation after $24 \mathrm{~h}$ ); unstable (phase separation or precipitate with $24 \mathrm{~h}$ ); clear (transparent or transparent with bluish ting) and non-clear (milky or turbid). The prepared formulations F1, F2, F6, F7, F8, F11, F12 and F13 was found to be clear after 24 h while F3, F4, F5, F9, F10, F14 and F15 become milky after $24 \mathrm{~h}$. In spite of the surfactant concentration, all the prepared formulations were observed to stable without any phase separation or precipitation of the drug.

\section{Refractive index}

The clarity of microemulsion could be estimated by measuring the refractive index of the formulations. In the present study, the refractive index of distilled water (standard) was used for comparison with the prepared formulations. It can be seen from the Table 2 the refractive index of the majority of the prepared formulations have similar values as that of distilled water $(1.3330 \pm 0.0002$ n.d.) at $28 \pm 0.5^{\circ} \mathrm{C}$. Whereas, the formulations F3, F4, F5, F9, F10, F14 and F15 showed some divergence when compared to that of standard and remaining formulations were found to be clear as water.

\section{$\mathrm{pH}$}

Stability of SEDDS formulations could be greatly affected by $\mathrm{pH}$. The change in the $\mathrm{pH}$ might affect the zeta potential of the formulation which in turn could affect the stability of preparation. ${ }^{22}$ All the prepared formulations showed similar $\mathrm{pH}$ values in the range of $6.20-6.73$ as shown in Table 2. Thus, stability of the formulation was not affected by $\mathrm{pH}$. It can be assumed that drug is not diffusing in the external phase and remains in the oil phase. Since, water is the external phase entire system showed $\mathrm{pH}$ of water. ${ }^{16}$

\section{Conductance}

The conductivity could be used to determine the type of microemulsion. It has been reported that this might be due to transition of oil microemulsion system to water microemulsion system. Thus it causes an increase of conductivity. ${ }^{22}$ In the present study, majority of prepared formulations indicated water continuously microemulsion system while, there was decreased in the values of few batches, and these may be due to the presence of oil globules. It can be observed from the results shown in Table 2 that higher values of conductivity were observed in Formulation F7 and F8 as compared to other formulations.

\section{$\%$ Transmittance}

The clarity of microemulsion can be observed by transparency, which can be measured in the form of $\%$ transmittance ( $\% \mathrm{~T})$. The prepared SEDDS was found to be $\mathrm{o} / \mathrm{w}$ type of microemulsion as the water was in the external phase. The prepared batches are showing higher $\% \mathrm{~T}$ greater than $96 \%$ was indicated by high clarity of microemulsion. While, the prepared batches having $\% \mathrm{~T}$ $<96 \%$ suggested less clarity of microemulsion. The lower values of $\% \mathrm{~T}$ could be due to large particle size of prepared microemulsions and the oil present in the system leads to a reduction of transparency. In the present study, formulation F8 showed highest transmittance compared to other formulations as shown in Table 2.

Table 2: Characterization of SEDDS formulations of rosuvastatin calcium

\begin{tabular}{|c|c|c|c|c|c|c|c|}
\hline Batches & $\begin{array}{l}\text { Refractive } \\
\text { index }\end{array}$ & $\mathrm{pH}$ & Conductance & $\begin{array}{c}\% \\
\text { transmittance }\end{array}$ & $\begin{array}{l}\text { Particle } \\
\text { size }\end{array}$ & $\begin{array}{c}\text { Zeta } \\
\text { potential }\end{array}$ & $\begin{array}{l}\% \text { drug } \\
\text { content }\end{array}$ \\
\hline$F_{1}$ & $1.3330 \pm 0.0003$ & $6.476 \pm 0.076$ & $96.13 \pm 0.61$ & $96.16 \pm 0.45$ & $106.3 \pm 2.2$ & -6.67 & $98.00 \pm 0.81$ \\
\hline $\mathrm{F}_{2}$ & $1.3331 \pm 0.0003$ & $6.570 \pm 0.040$ & $95.16 \pm 0.55$ & $89.73 \pm 0.51$ & $88.12 \pm 1.7$ & -10.28 & $97.63 \pm 0.85$ \\
\hline $\mathrm{F}_{3}$ & $1.3356 \pm 0.0005$ & $6.393 \pm 0.041$ & $93.30 \pm 0.65$ & $86.13 \pm 0.37$ & $77.82 \pm 3.4$ & -12.11 & $96.90 \pm 0.66$ \\
\hline $\mathrm{F}_{4}$ & $1.3363 \pm 0.0005$ & $6.546 \pm 0.040$ & $91.90 \pm 0.65$ & $81.26 \pm 0.35$ & $83.14 \pm 1.4$ & -9.45 & $97.60 \pm 0.87$ \\
\hline $\mathrm{F}_{5}$ & $1.3366 \pm 0.0005$ & $6.693 \pm 0.032$ & $91.03 \pm 0.35$ & $79.06 \pm 0.61$ & $66.24 \pm 1.5$ & -7.54 & $97.66 \pm 0.90$ \\
\hline $\mathrm{F}_{6}$ & $1.3332 \pm 0.0002$ & $6.583 \pm 0.087$ & $96.50 \pm 0.62$ & $97.23 \pm 0.65$ & $53.71 \pm 2.1$ & -14.72 & $98.13 \pm 0.35$ \\
\hline $\mathrm{F}_{7}$ & $1.3333 \pm 0.0003$ & $6.546 \pm 0.065$ & $97.10 \pm 0.91$ & $97.13 \pm 0.35$ & $26.93 \pm 1.6$ & -18.35 & $97.26 \pm 0.65$ \\
\hline $\mathrm{F}_{8}$ & $1.3332 \pm 0.0002$ & $6.586 \pm 0.061$ & $98.70 \pm 0.40$ & $98.56 \pm 0.25$ & $10.59 \pm 1.1$ & -22.11 & $98.36 \pm 0.58$ \\
\hline $\mathrm{F}_{9}$ & $1.3348 \pm 0.0004$ & $6.426 \pm 0.065$ & $94.86 \pm 0.066$ & $92.33 \pm 0.45$ & $74.21 \pm 1.8$ & -15.23 & $97.60 \pm 0.45$ \\
\hline$F_{10}$ & $1.3359 \pm 0.0002$ & $6.870 \pm 0.030$ & $92.70 \pm 0.30$ & $86.30 \pm 0.36$ & $68.74 \pm 1.7$ & -10.22 & $97.53 \pm 0.70$ \\
\hline$F_{11}$ & $1.3333 \pm 0.0003$ & $6.720 \pm 0.036$ & $96.13 \pm 0.75$ & $97.26 \pm 0.35$ & $67.49 \pm 2.2$ & -12.86 & $96.76 \pm 0.60$ \\
\hline$F_{12}$ & $1.3334 \pm 0.0002$ & $6.673 \pm 0.047$ & $96.03 \pm 0.35$ & $98.46 \pm 0.11$ & $45.26 \pm 1.6$ & -16.42 & $97.83 \pm 0.65$ \\
\hline $\mathrm{F}_{13}$ & $1.3333 \pm 0.0002$ & $6.896 \pm 0.041$ & $96.16 \pm 0.55$ & $92.00 \pm 0.65$ & $33.68 \pm 0.8$ & -13.89 & $98.16 \pm 0.37$ \\
\hline $\mathrm{F}_{14}$ & $1.3341 \pm 0.0004$ & $6.706 \pm 0.033$ & $94.70 \pm 0.36$ & $87.86 \pm 0.60$ & $62.31 \pm 1.3$ & -14.38 & $97.10 \pm 0.75$ \\
\hline $\mathrm{F}_{15}$ & $1.3362 \pm 000.5$ & $6.840 \pm 0.036$ & $91.36 \pm 0.77$ & $84.93 \pm 0.41$ & $45.12 \pm 1.2$ & -9.54 & $97.76 \pm 0.40$ \\
\hline
\end{tabular}




\section{Particle size and zeta potential analysis}

The rate and extent of drug release and absorption could be dependent on the globule size which is a critical parameter in self-emulsification process. It has also been reported that the smaller particle size of the emulsion droplets may lead to more rapid absorption and improve the bioavailability. ${ }^{23}$ The degree of attraction or repulsion between particles could be measured by zeta potential. In addition, the particles present in the polar medium like water would possess a surface charge. The blank SEEDS formulation exhibited almost no charge whereas a negative charge was obtained with drug-loaded SEDDS. This could be because the emulsifier used in the formulation, which is a nonionicsurfactant. ${ }^{24,25}$ It can be observed from the results shown in Table 2 that the smallest particle size $(10.59 \mathrm{~nm})$ and lowest zeta potential $(-22.11 \mathrm{mV})$ was observed in Formulation F8 as compared to other batches.

\section{TEM}

The TEM operates at the same basic principal as the light microscope but uses electrons instead of light. The TEM was used to examine the morphology of prepared microemulsion. Figure 3 depicts spherical shaped globules of prepared batch F8 in addition the droplets were dark within bright surroundings.

\section{Characterization of S-SEDDS}

\section{Visual observation}

The clarity and phase separation of drug were examined of the prepared formulations for a period of 24 has described in Characterization of SEDDS. It was observed that prepared formulations F1, F2, F6, F7, F8, F11, F12 and F13 was found to be clear after $24 \mathrm{~h}$ while F3, F4, F5, F9, F10, F14 and F15 become milky after $24 \mathrm{~h}$ independent to the amount of surfactant. Further,

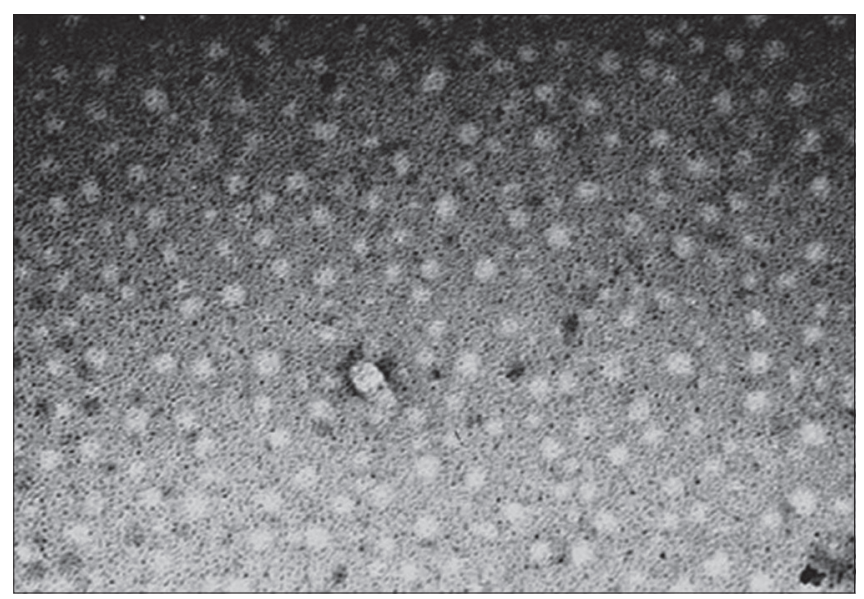

Figure 3: Transmission electron microscopy image of Batch F8 no sign of drug precipitation and phase separation were observed.

\section{Robustness on dilution}

Any sign of precipitation or phase separation were not observed in diluted S-SEDDS on storage in the different dilution media like distilled water, phosphate buffer $\mathrm{pH} 6.8$ and $0.1 \mathrm{~N} \mathrm{HCl}$. In the present study, formulations F1, F2, F6, F7, F8, F11, F12 and F13 did not show any cloudiness, phase separation or precipitation for $24 \mathrm{~h}$ while, instantaneously turbid dispersion formed with 100-1000 fold dilutions of Formulations F3, F4, F5, F9, F10, F14 and F15. The robustness of prepared formulations (F1, F2, F6, F7, F8, F11, F12 and F13) could be confirmed due to its clarity in different dilution volumes of various media. Whereas, in other formulations (F3, F4, F5, F9, F10, F14 and F15) loss of clarity was observed these might be due to a higher amount of oil in it.

\section{Drug content}

The UV spectrophotometric method was used to determine the drug content of ROS S-SEDDS and it was estimated considering $10 \mathrm{mg}$ of ROS as $100 \%$. In the present investigation, the prepared formulations were within the specified limit (90-110\%) of drug content according to IP 2010. Drug content of all the S-SEDDS formulations is shown in Table 2.

\section{Differential scanning colorimetry}

The quantitative and qualitative information about the physical state of drug present inside S-SEDDS could be studied using it's thermal behavior by DSC. The thermogram of pure drug (ROS) and optimized formulation (F8) were recorded. It can be seen from Figure 4 that no endotherm was present because the drug was present either molecularly dispersed or as a solid solution in the S-SEDDS. The pure drug (ROS) indicated sharp peak at $125.66^{\circ} \mathrm{C}$ which could be due to its melting point while, no characteristic peak was observed at the same temperature indicating the drug might be dispersed in the molecular matrix.

\section{FTIR study}

The possibility of any interaction between the drug and other formulation ingredients could be identified by recording the Infrared (IR) spectra of ROS and selected S-SEDDS were recorded. IR spectra of pure ROS and prepared S-SEDDS are shown in Figure 5. FTIR spectra demonstrated the evidence that the characteristics peaks of different function group of ROS in prepared S-SEDDS were not much deviate from the peaks of pure drug. Thus, the results suggested that there is no possible interaction between drug and excipients used in this study. 

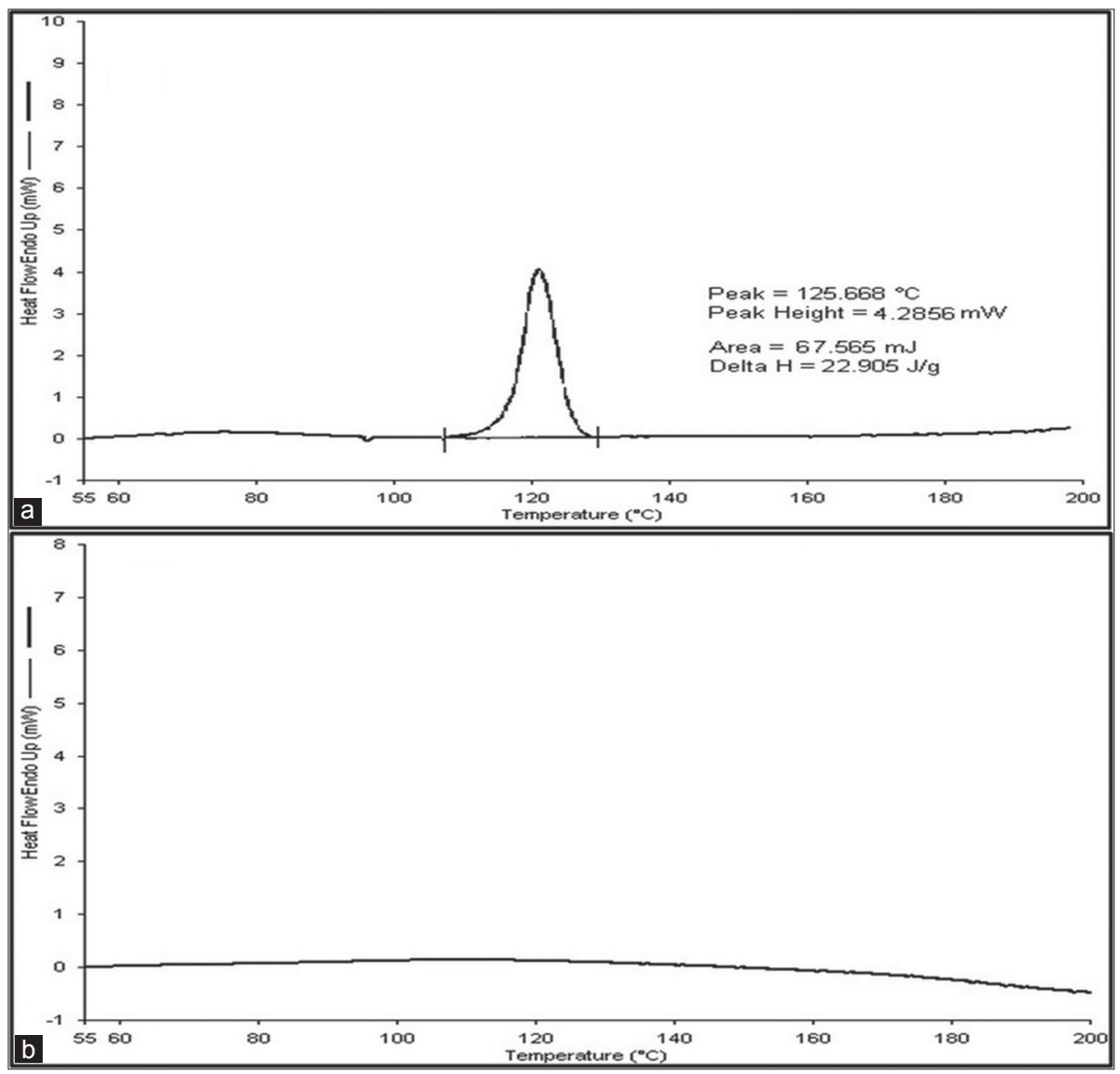

Figure 4: (a) Differential scanning colorimetry (DSC) thermogram of rosuvastatin calcium and (B) DSC thermogram prepared Batch F8

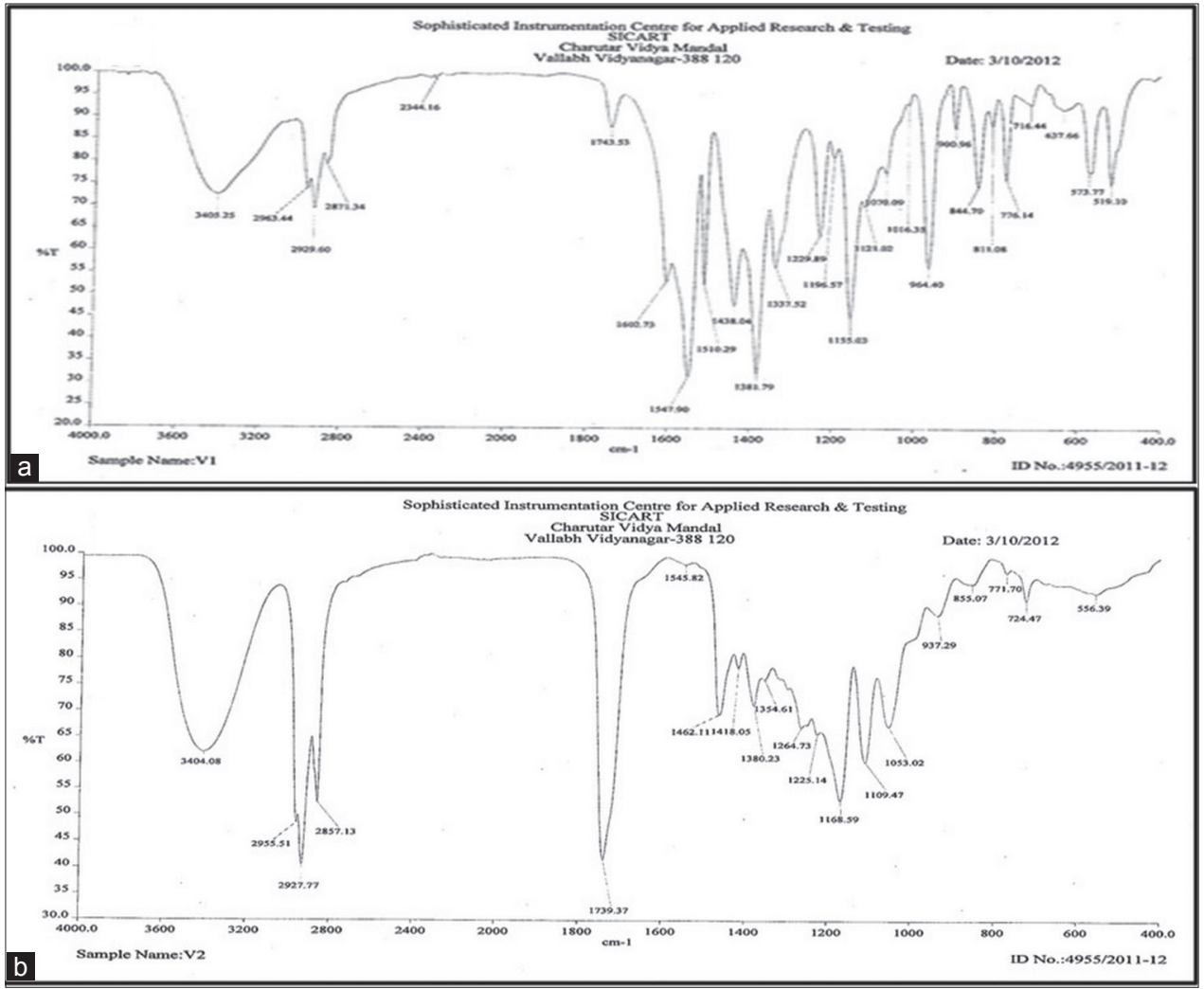

Figure 5: (a) Infrared (IR) spectra of rosuvastatin calcium and (b) IR spectra of Batch F8 


\section{In vitro dissolution rate studies}

The behavior of drug transport through GI tract could be predicted by studying the In vitro dissolution of prepared formulations. Dissolution profile of pure drug (ROS) and prepared S-SEDDS formulations were evaluated in phosphate buffer $\mathrm{pH}$ 6.8. The comparative in vitro release of ROS and the prepared batches of S-SEDDS is shown in Figure 6. Cumulative \% drug release of prepared batches was found to be in the range of 70\%-97\% while that of plain ROS was found to be $53 \%$. These results are in agreement with the previous findings explaining that the SEDDS formulation, which leads to the spontaneous formation of a microemulsion having a smaller droplet size and it leads to a faster release of drug into the aqueous environment as compared with that of pure drug. ${ }^{14,15}$ In the present study, the highest release rate $(97.8 \%)$ was observed in batch F8 compared to other batches as shown in Figure 6.

The batch F8 demonstrated satisfactory drug content, particle size, zeta potential and drug release as compared to other prepared batches. Hence, batch F8 was considered to be as optimized S-SEDDS formulation. The dissolution profile of the prepared formulation (F8)

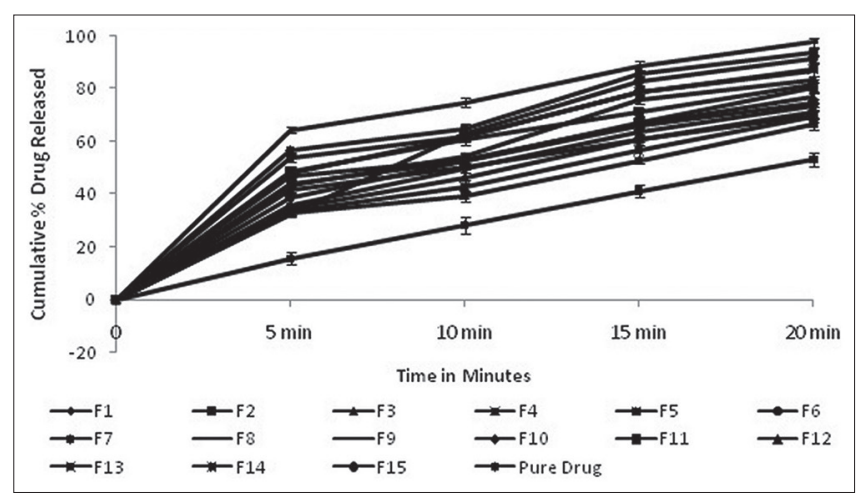

Figure 6: Dissolution profile of prepared solid self-emulsifying drug delivery system formulation and pure rosuvastatin calcium (values are mean \pm standard deviation; $\mathrm{n}=3$ )

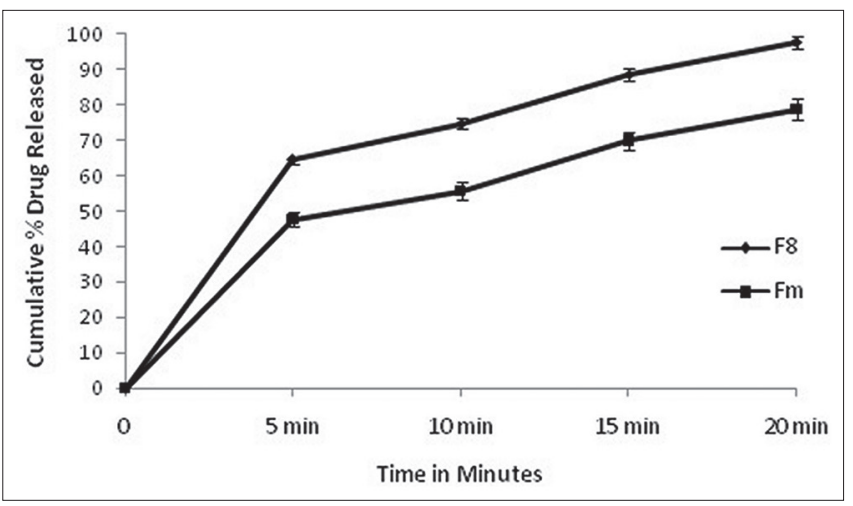

Figure 7: Comparative dissolution profile of Batch F8 with marketed product (values are mean \pm standard deviation; $n=3$ ) of ROS S-SEDDS was compared with marketed product (Fm). The comparative drug release is shown in Figure 7 indicates that the selected batch exhibited higher release as compared to marketed formulation. These findings could predict an enhancement of absorption and, as a result, the bioavailability due to improved dissolution of ROS. ${ }^{16}$

\section{CONCLUSION}

ROS S-SEDDS consisting of the oil phase (capmul MCM) of $20 \%$, surfactant (cremophor ELP) of $53.33 \%$ and co-surfactant (propylene glycol) of $26.66 \%$ was found as optimized formulation. The resultant microemulsion was negatively charged with smaller particle size. The drug release of ROS from S-SEDDS was faster than the marketed formulation suggesting that this dosage form might be an alternate for slightly water soluble drug. In the future, a human study after oral administration of ROS S-SEDDS is also required for its clinical use.

\section{ACKNOWLEDGMENTS}

The authors are thankful to Pravah Lab Pvt. Ltd. Hyderabad, India for the gift sample of ROS pure powder, Sophisticated Instrumentation Center for Applied Research and Testing (SICART) (Vallabh Vidyanagar, India) for providing facilities for carrying out analytical work, Gattefosse (Saint-Priest, France), Abitec Corporation (Janesville, USA), BASF (Mumbai, India), Sasol (Witten, Germany) for providing gratis samples of excipients.

\section{REFERENCES}

1. Kommuru TR, Gurley B, Khan MA, Reddy IK. Self-emulsifying drug delivery systems (SEDDS) of coenzyme Q10: Formulation development and bioavailability assessment. Int J Pharm 2001;212:233-46.

2. Katteboina S, Chandrasekhar VS, Balaji S. Approaches for the development of solid self-emulsifying drug delivery systems and dosage forms. Asian J Pharm Sci 2009;4:240-53.

3. Humberstone AJ, Charman WN. Lipid-based vehicles for the oral delivery of poorly water soluble drugs. Adv Drug Deliv Rev 1997;25:103-28.

4. Pouton CW. Formulation of self-emulsifying drug delivery systems. Adv Drug Deliv Rev 1997;25:47-58.

5. Gursoy RN, Benita S. Self-emulsifying drug delivery systems (SEDDS) for improved oral delivery of lipophilic drugs. Biomed Pharmacother 2004;58:173-82.

6. Bhagwat DA, D'Souza JI. Formulation and evaluation of solid self micro emulsifying drug delivery system using aerosil 200 as solid carrier. Int Curr Pharm J 2012;1:414-9.

7. Agarwal V, Siddiqui A, Ali H, Nazzal S. Dissolution and powder flow characterization of solid self-emulsified drug delivery system (SEDDS). Int J Pharm 2009;366:44-52.

8. Balakrishnan P, Lee BJ, Oh DH, Kim JO, Hong MJ, Jee JP, et al. Enhanced oral bioavailability of dexibuprofen by a novel solid self-emulsifying drug delivery system (SEDDS). Eur J Pharm Biopharm 2009;72:539-45. 
9. Shek A, Ferrill MJ. Statin-fibrate combination therapy. Ann Pharmacother 2001;35:908-17.

10. Blatter Fritz, Martin Szelagiewicz, Paul Adriaan Van Der Schaaf. Crystalline forms of Rosuvastatin calcium salt. United States Patent US 7932387. 2011 April 26.

11. Date AA, Nagarsenker MS. Design and evaluation of self-nanoemulsifying drug delivery systems (SNEDDS) for cefpodoxime proxetil. Int J Pharm 2007;329:166-72.

12. Thimmaraju MK, Ramagiri V, Bheemanapally K, Bojja S, Kola V, Nerella R, et al. Preparation and characterization of rosuvastatin calcium nanoemulsions. Lat Am J Pharm 2013;32:1445-51.

13. Azeem A, Rizwan M, Ahmad FJ, Iqbal Z, Khar RK, Aqil M, et al. Nanoemulsion components screening and selection: A technical note. AAPS PharmSciTech 2009;10:69-76.

14. Patel AR, Vavia PR. Preparation and in vivo evaluation of SMEDDS (selfmicroemulsifying drug delivery system) containing fenofibrate. AAPS J 2007;9:E344-52

15. Trivedi KK, Patel PV, Pujara Z. Development and characterization of liquid and solid selfemulsifying drug delivery system of fexofenadine. J Pharm Invest 2013;43:385-94

16. Singh AK, Chaurasiya A, Awasthi A, Mishra G, Asati D, Khar RK, et al. Oral bioavailability enhancement of exemestane from selfmicroemulsifying drug delivery system (SMEDDS). AAPS PharmSciTech 2009;10:906-16
17. Yosara SR, Magda A. Self nanoemulsifying drug delivery systems of temoxifen citrate: Design and optimization. Int J Pharm 2009;380:133-41.

18. Yan YD, Kim JA, Kwak MK, Yoo BK, Yong CS, Choi HG. Enhanced oral bioavailability of curcumin via a solid lipid-based self-emulsifying drug delivery system using a spray-drying technique. Biol Pharm Bul 2011;34:1179-86

19. Shaji J, Joshi V. Self microemulsifying drug delivery system (SMEDDS) for improvement of bioavailability of hydrophilic drugs and its potentia to give sustain release dosage form. Indian J Pharm Educ 2005;39:130-5.

20. Shah NH, Carvajal MT, Patel CI, Infeld MH, Malick AW. Self-emulsifying drug delivery systems (SEDDS) with polyglycolized glycerides for improving in vitro dissolution and oral absorption of lipophilic drugs. Int J Pharm 1994;106:15-23.

21. Thakkar H, Nangesh J, Parmar M, Patel D. Formulation and characterization of lipid-based drug delivery system of raloxifene-microemulsion and selfmicroemulsifying drug delivery system. J Pharm Bioallied Sci 2011;3:442-8.

22. Subramanian N, Ray S, Ghosal SK, Bhadra R, Moulik SP. Formulation design of self-microemulsifying drug delivery systems for improved oral bioavailability of celecoxib. Biol Pharm Bull 2004;27:1993-9.

23. Prajapati ST, Joshi HA, Patel CN. Preparation and characterization of self-microemulsifying drug delivery system of olmesartan medoxomil for bioavailability improvement. J Pharm 2013;2013:1-9.

24. Jeevana JB, Sreelakshmi K. Design and evaluation of self-nanoemulsifying drug delivery system of flutamide. J Young Pharm 2011;3:4-8. 